\section{Atoms in Optical Lattices}

\author{
J.Y. Courtois, G. Grynberg \\ Laboratoire Kastler-Brossel \\ Département de Physique de l'École Normale Supérieure, Paris
}

\section{Recent fundamental results demonstrate that it should be possible to probe concepts developed for condensed matter at totally different scales using optical lattices, where the defect-free confining potential is created by light interference.}

During the last few months, several groups have shown that ordered, dilute atomic media where atoms bound by light essentially oscillate around their equilibrium positions display novel properties. The results imply a dramatic reappraisal of the way one views the gaseous state. The typical distance between the atoms in these light-induced lattices is of the order of the optical wavelength, i.e., one micron, and their realisation was made possible by techniques to laser cool neutral atoms to temperatures in the microkelvin range. Ever since Hänsch and Schawlow first proposed laser cooling in 1975, its development as well as our understanding of cold atoms have mainly resulted from successive breakthroughs, often motivated by unexpected results which defied the predictions of generally accepted theories.

The principle of laser cooling is as follows: an atom loses kinetic energy on interacting with several laser beams tuned near a transition connecting the atom's ground-state to one of its excited states provided the photons absorbed in the laser waves (which bring the atom to the excited state) have an average energy below that of the fluorescence photons emitted in the atomic de-excitation process. In 1988, it was usually assumed that the minimum temperature that could be achieved by laser-cooling atoms was determined by the natural width of the excited state involved in the cooling process, i.e., of the order of a few $\mathrm{mK}$. However, Phillips and coworkers at the National Institute of Science and Technology (NIST) near Washington DC, showed that it was in fact possible to cool atoms far beyond this limit down to temperatures in the microKelvin range. This observation was eventually

Jean-Yves Courtoise obtained his $\mathrm{PhD}$ from the École Normale Supérieure, Paris, in 1993 where he now works.

Gilbert Grynberg is Professor of Physics at the ENS, Paris. He received his PhD in 1976 from the University of Paris and he has coathored two books dealing with atom-photon interactions. interpreted in terms of an approach, developed independently by Cohen-Tannoudji and Dalibard in Paris and by Chu and coworkers in Stanford, which accounted for the fact that the ground level of the atomic transitions used in the experiments had an internal structure consisting of several magnetic sub-levels [1].

\section{Sisyphus Cooling}

In order to illustrate the cooling mechanism, which is now known as Sisyphus cooling after the well-known legend in Greek mythology, we restrict ourselves to the simple case of a onedimensional geometry. Consider an atom for which the angular momentum $J_{\mathrm{a}}$ of the ground-state is equal to $1 / 2$. This ground-

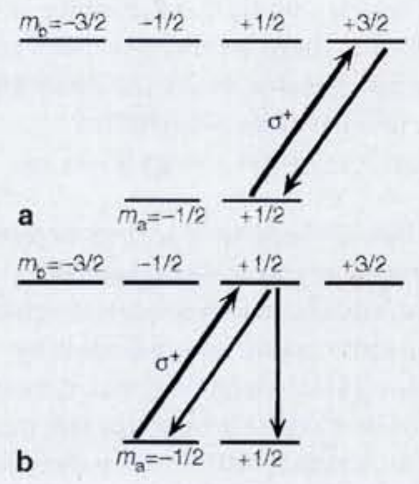

Fig. 1a-c. Optical pumping and light-shifts. Consider an atom with a ground-state of angular momentum $J_{9}=1 / 2$, which interacts with a laser beam tuned close to an atomic transition connecting the ground-state to an excited state of angular momentum $J_{\mathrm{e}}=3 / 2$. $A \sigma^{+}$polarized laser wave carries quanta of angular momentum along the propagation direction $+z$ equal to $+h$ per photon. It follows that light absorption by an atom in the ground-state sub-level $m_{\mathrm{a}}=+1 / 2$ brings the atom to the excited-state sub-level $m_{b}=+3 / 2$, from which it can only decay towards the initial state (a). By contrast, light absorption by an atom in the $m_{\mathrm{a}}=-1 / 2$ sublevel brings the atom to the Zeeman sub-level $m_{\mathrm{b}}=-1 / 2$, from which it can decay towards either the $m_{\mathrm{a}}=-1 / 2$ or $m_{\mathrm{a}}=+1 / 2$ sublevels $(\mathbf{b})$ because the selection rule for spontaneous emission is $\left|m_{b}-m_{a}\right|$. Hence, the absorption-spontaneous emission cycles state actually consists of two Zeeman sublevels identified by the magnetic quantum numbers $m_{\mathrm{a}}=-1 / 2$ and $m_{\mathrm{a}}=+1 / 2$ (see Fig. 1). It can be coupled to an excited state of angular momentum $J_{b}=3 / 2$ by a quasi-resonant light field resulting from two counter-propagating laser beams having the same wavelength $\lambda$ but with crossed linear polarizations. The superimposition of these beams leads to a standing wave in which the polarization changes at each point in space with a periodicity $\lambda / 2$. As illustrated in Fig. 2, depending on the relative (spacedependent) phase between the incident waves, the polarization of the resulting field is linear, elliptical or circular (the circular polarization is denoted by the symbols $\sigma^{+}$or $\sigma^{-}$depending on the direction of rotation of the electric field).

The laser field has two essential effects on the Zeeman sublevels $m_{\mathrm{a}}=-1 / 2$ and $m_{\mathrm{a}}=+1 / 2$ (see Fig. 1). First, it modifies the relative population of these sublevels by an amount that depends on the local polarization of the electric field (this is the optical pumping process predicted by Kastler in 1950). In particular, at points of circular polarization, the light field tends to "pump" the atoms into a single sublevel

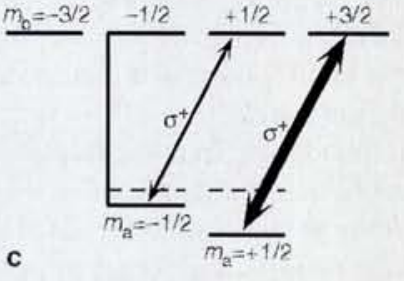

tend to accumulate the atoms in the $m_{\mathrm{a}}=+1 / 2$ state. This is the optical pumping process induced by $\sigma^{+}$ polarized light.

$\mathrm{A} \sigma^{+}$polarized incident wave not only affects the relative populations of the $m_{\mathrm{a}}=-1 / 2$ and

$m_{\mathrm{a}}=+1 / 2$ sublevels, but also induces different energy shifts (called light-shifts) of these levels. This is because the matrix elements of the electric dipole operator between the states $m_{\mathrm{a}}=-1 / 2$ and $m_{\mathrm{b}}=+1 / 2$ on one hand, and $m_{\mathrm{a}}=+1 / 2$ and $m_{\mathrm{b}}=+3 / 2$ on the other hand, are not equal. Since the repulsion of the levels due to the light-induced coupling is proportional to the square of these matrix elements, the $m_{a}=+1 / 2$ sublevel is more light-shifted than the $m_{\mathrm{a}}=-1 / 2$ state, because it corresponds to the largest matrix element (c). As a result, the $m_{\mathrm{a}}=+1 / 2$ state has the lowest energy and the largest population when $\omega<\omega_{\mathrm{A}}$. 
( $m_{\mathrm{a}}=+1 / 2$ for a $\sigma^{+}$polarized field, $m_{\mathrm{a}}=-1 / 2$ for $\mathrm{a} \sigma$ polarized field).

Second, the sublevels generally experience different energy shifts (these are the light-shifts discovered by CohenTannoudji in 1961). The magnitude of this effect depends on the local polarization of the electric field, and on the difference between the laser frequency $\omega$ and the atomic resonance frequency $\omega_{\mathrm{A}}$. In particular, the latter determines the sign of the level shifts (negative for $\omega<\omega_{A}$, positive for $\omega>\omega_{A}$ ), whereas the former governs the relative energy shifts of the levels. For example, one can show that the light-shift of the sublevel $m_{\mathrm{a}}=+1 / 2$ is a maximum - and the shift for sublevel $m_{\mathrm{a}}=-1 / 2$ a minimum at points where the light is $\sigma^{+}$polarized; the inverse is the case at points of $\sigma$ polarization.

Thus, the combined action of optical pumping and light-shifts tends to accumulate the atoms in the sublevel having the largest light-shift. In particular, in the case where the laser frequency is chosen such that $\omega<\omega_{\mathrm{A}}$ (negative lightshifts), atoms are preferentially pumped into the Zeeman sublevel having the lowest energy. Moreover, the continuous spatial variation of the field polarization leads to a spatial modulation of the light-shifted energies of the ground-state sublevels, which act as an external potential for the variables associated with the dynamics of the atomic centre-of-mass (Fig. 2). This situation reminds one of molecular physics where the nuclei potential is determined by the energy of the electrons. However, the magnitudes of the effects are dramatically different. In the case of molecules, the depth of the potential wells is related to the Coulomb potential, and is typically (in units of temperatures) of the order of $10^{4} \mathrm{~K}$. Molecules are usually stable as long as the external temperature remains small compared to this potential depth. In the case of the potential wells associated with the light-shifts illustrated in Fig. 2, the situation is completely different because the potential depth is only of the order of $10^{-4} \mathrm{~K}$. Ultra-low temperatures in the millikelvin range are therefore required in order to trap atoms for a long time in laserinduced optical potential wells.

One needs to consider a moving atom and follow the time evolution of its velocity in order to understand the mechanism leading to atomic temperatures compatible with trapping in the optical potential wells. Owing to the spatial modulation of the optical potential, the atom behaves like a marble on a corrugated surface: it decelerates or accelerates when going up or down a potential hill, because its total mechanical energy (sum of the kinetic and the potential energies) is a constant (Fig. $2 b)$. However, because of the optical pumping, the atomic motion is slightly more subtle. The atom indeed decelerates as it climbs the potential hill associated with its magnetic sub-state, but at the top of the hill, instead of being accelerated towards the next hill, it is optically pumped (because of the local field polarization) to the other Zeeman sublevel having a minimum potential energy at this location, with the excess energy being removed by the fluorescence photon. The process is repeated, with the atom climbing successive hills and undergoing successive decelerations. Cooling proceeds until the atom has insufficient energy to climb a potential hill, at which point it is trapped in a well.

Once the atom is trapped, classically it oscillates at the bottom of the well at a frequency $\Omega_{\mathrm{v}}$ depending on the curvature of the potential energy field and on the atomic mass. Because the spatial extent of the potential well is less than the optical wavelength $\lambda$, the amplitude of the atomic oscillations at the bottom of the well can be much smaller than $\lambda$. In a quantum mechanical description of the centre-ofmass motion, there is a discrete number of bound vibrational levels inside a potential well. As in most cases of quantum oscillators, the lowest energy levels are

Fig. 2. a: The superposition of two counter-propagating laser beams having the same wavelength $\lambda$ and orthogonal polarizations gives rise to a standing wave for which the polarization is space-dependent. The polarization is alternatively circular $\sigma$ in $z=0$, linear in $z=\lambda / 8$, circular $\sigma^{+}$in $z=\lambda / 4$, linear in $z=3 \lambda / 8$, etc., with a spatial periodicity $\lambda / 2$. This polarization gradient leads to a periodic spatial modulation of the light-shifts of the Zeeman sublevels $m_{\mathrm{a}}=1 / 2$ and $m_{\mathrm{a}}=-1 / 2$, which acts as a potential for the motion of the atomic centreof-mass. Note that the potential is different for each of the Zeeman sublevels. The quantized levels of the atomic motion near the bottom of the potential wells are separated by one quantum of vibration.

b: Sisyphus cooling mechanism. An atom located in the $m_{\mathrm{a}}=-1 / 2$ sublevel, having velocity $v$ and propagating in the $+z$ direction climbs a potential hill. Near the peak, the light polarization is $\sigma^{+}$and the atom is optically pumped in the $m_{\mathrm{a}}=1 / 2$ sublevel. The atom thus returns close to a minimum of the potential, and the climbing/ optical pumping sequences proceed. Each time it climbs a potential hill, the atom loses a fraction of its kinetic energy until its velocity is no longer sufficient to reach the next peak. The atom is then trapped in a potential well. given by the usual expression $(n+1 / 2) h \Omega_{\mathrm{v}}$, where $n$ is a positive integer, and $h$ is the Planck constant. In fact, the temperature is so low that only the lowest energy levels of the potential wells are populated (Fig. 2).

Castin and Dalibard [2] have derived the steady-state populations of the different energy levels as a function of the intensity and frequency detuning of the cooling beams. They showed that a significant fraction, of the order of one-quarter of the atoms, could be accumulated in the lowest $n=0$ energy level, for which the spatial extent of the wavefunction is typically $\lambda / 30$.

The Sisyphus cooling mechanism is actually much more general than what might be inferred from the simplified model. It can be transposed to any atomic transition between a ground-state of angular momentum $J_{\mathrm{g}}>0$ and an excited state of angular momentum $J_{\mathrm{e}}$. It also plays a crucial role in most of the two- and threedimensional geometries.

\section{Quantized Atomic Motion}

Observing the quantized energy levels in the wells, while fascinating, appeared to be impossible. Vibrational states have a width that is related to the time/energy Heisenberg uncertainty principle: if the lifetime of an atom in a quantum vibrational state $n$ is $\tau_{n}$, the width of the level is of the order of $h / \tau_{n}$. Owing to the absorption-fluorescence cycles described in Fig. 2b, atoms excited by the laser beams leave a given sublevel after an average time
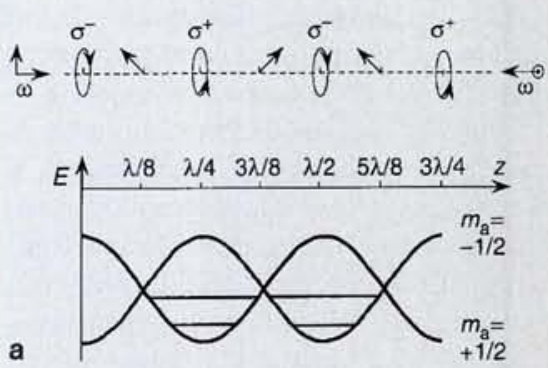

b

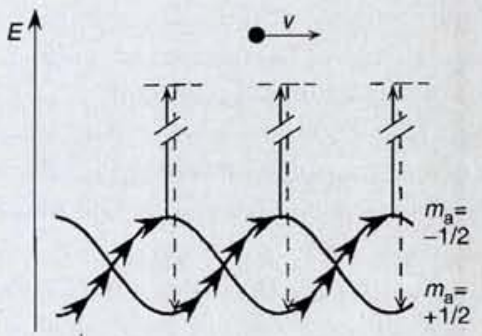


equal to the optical pumping time $\tau_{\mathrm{p}}$ which, in typical experimental conditions, is close to or even larger than the distance $h \Omega_{v}$ between the vibrational states. As a result, the different energy levels overlap each other. However, the width is overestimated when the light polarization at the bottom of the potential wells is purely circular. We have shown that the atoms actually leave a given sublevel after a time $\tau_{p}$, but the fluorescence process essentially returns them to their original sublevel. This characteristic is more pronounced the deeper the potential wells. So the actual lifetime to be used in estimating the widths of the vibrational levels is the time after an atom leaves the vibrational level, which is much longer than $\tau_{p}[3]$. The width of the lowest quantized vibrational states is therefore generally much smaller than their separation, so the states can be observed.

The first experimental demonstration of the quantization of atomic motion in the potential of Fig. 2 was performed in Paris in 1992 [4]. The experiment was based on the observation of stimulated Raman transitions between vibrational levels using probe-absorption spectroscopy. A very low intensity probe beam was superimposed on the two laser beams to create the optical potential of Fig. 2 . The frequency $\omega$ ' of the probe beam could be varied continuously around the lattice frequency $\omega$. If the population of the vibrational state $n=0$ is larger than that of the $n=1$ state, a resonant population transfer occurs between these levels when the difference between the energy quanta associated with the lattice and the probe waves is equal to the vibrational energy quantum, i.e., when $\left|\omega-\omega^{\prime}\right|=\Omega_{v}$ (the stimulated Raman effect). When $\omega-\omega^{\prime}=$ $+\Omega_{v}$, energy flows from the waves creating the optical potential (which undergo absorption) towards the probe beam, which is amplified. Conversely, if $\omega-\omega^{\prime}=$ $-\Omega_{v}$, the probe beam is absorbed (Fig. 3 b).

The experimental spectrum giving the variation of the probe intensity after transmission through the atomic medium revealed resonant amplification of the probe beam for $\omega-\omega^{\prime}=+\Omega_{v}$, and resonant absorption for $\omega-\omega^{\prime}=-\Omega_{v}$ (Fig. 3c). The spectrum's characteristics (positions and widths of the resonances) confirm the existence of vibration at the bottom of the potential wells, and of a significant fraction of atoms being in the lowest energy states.

Soon after this observation, the NIST group demonstrated quantization of atomic motion using spontaneous Raman

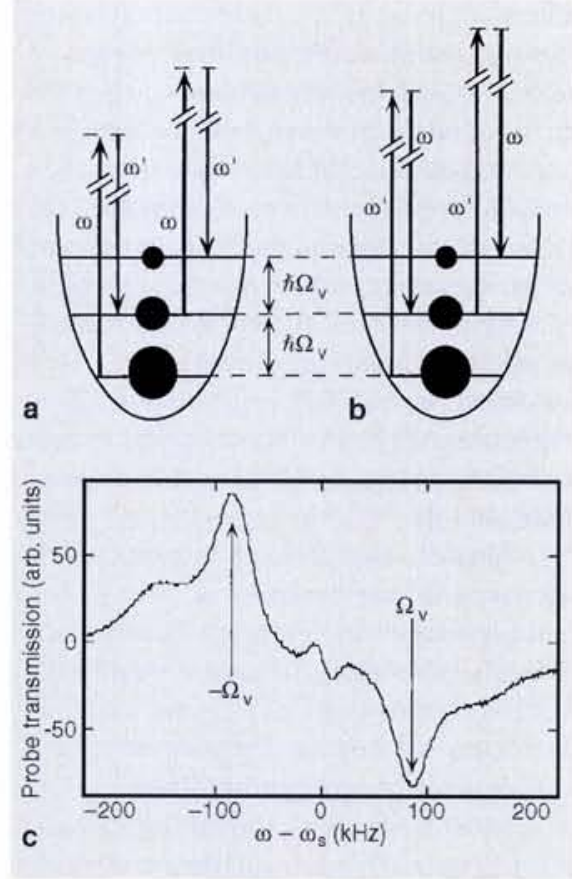

Fig. 3. Stimulated Raman transition between vibrational levels. a: When the probe wave frequency $\omega^{\prime}$ is smaller than the frequency $\omega$ of the lattice beams (the laser beams creating the optical potential) by an amount equal to the vibration quantum $\Omega_{\mathrm{y}}$, a resonant population transfer occurs from the highly populated levels to the levels having the lowest population (in the figure, the population is proportional to the dimension of the black dot). This population transfer manifests itself as an amplification of the probe (stimulated Raman gain).

b: The reverse process when the probe beam frequency $\omega$ ' is larger than $\omega$. The population transfer is now associated with absorption in the probe beam. c: Experimentally recorded probe transmission spectrum for caesium atoms showing two Raman peaks: one in absorption for $\omega^{\prime}-\omega=\Omega_{v}$, and one in amplification for $\omega^{\prime}-\omega=\Omega_{\mathrm{y}}$ (the quantum of vibration $\Omega_{\mathrm{v}}$ is equal to $90 \mathrm{kHz}$ ).

scattering [5]. This method, which only involves lattice beams of frequency $\omega$, is based on analysing the frequency spectrum of the fluorescence spontaneously emitted by the atoms. After absorption of a laser photon, an atom in the $n=0$ vibrational level returns towards the fundamental electronic state by emitting either a photon having the same frequency (Rayleigh scattering), or a photon of frequency $\omega-\Omega_{\mathrm{v}}$ (Raman scattering). An absorption/fluorescence cycle for an atom in the $n=1$ vibrational level leads to Rayleigh and Raman photons having the same frequencies (corresponding to a simple translation of the initial vibrational state of the scattering process), and also to Raman photons of frequency $\omega+\Omega_{v}$ when the final state of the process is the $n=0$ level. The resonances centred around $\omega-\Omega_{v}$ and $\omega-\Omega_{v}$ have different amplitudes because the former involves all vibrational levels, whereas the $n=0$ state does not contribute to the latter. This characteristic, which was also confirmed experimentally, allows one to estimate the fraction of atoms occupying the $n=0$ level as well as the atomic temperature (of the order of $5 \mathrm{mK}$ ). The Rayleigh line (centred on $\omega$ ) was much more intense than the Raman lines, confirming that after an absorption/fluorescence cycle, most of the atoms returned towards the vibrational level they left.

\section{An Ordered Antiferromagnet}

As shown in Fig.2, light polarization at the minima of the optical potential wells involved in the Sisyphus cooling scheme is circular and alternatively $\sigma^{+}$or $\sigma^{-}$. The distance between two wells corresponding to opposite circular polarization is $(2 p+1) \lambda / 4$ where $p$ is an integer. At points where the light is $\sigma^{+}$circularly polarized, optical pumping transfers the atoms in the lower vibrational states of the potential corresponding to $m_{\mathrm{a}}=+1 / 2$. Conversely, when the $\sigma$ polarization is dominant, optical pumping transports the atoms towards the $m_{\mathrm{a}}=-1 / 2$ substate. In fact, the quantum number $m_{\mathrm{a}}$ is associated with the projection of the atomic magnetic moment along the $z$-direction. Hence, negative and positive values such as $m_{\mathrm{a}}=+1 / 2$ and $m_{\mathrm{a}}=-1 / 2$ correspond to opposite directions for the magnetic moment. Atoms can thus be trapped in potential wells in such a way that their magnetic moments point either in the $+z$ direction or in the $-z$ direction. Such a structure resembles an antiferromagnetic crystal. There is, however, a fundamental difference: whilst in solid-state physics, the order and the directions of the magnetic moment result from interatomic interaction, in an optical lattice the order is imposed by light.

A one-dimensional optical lattice is not necessarily antiferromagnetic. An optical potential in which all the bound atoms have a magnetic moment pointing in the same direction has been studied experimentally [6] using two counterpropagating beams of the same circular polarization, plus a transverse magnetic field (an alternative method of Sisyphus cooling). In this example of a ferro- 
magnetic optical lattice, the direction of the magnetic moment is imposed by the circular polarization of light.

\section{Two-dimensional Lattices}

The structures discussed up to now are one-dimensional, being only realised in a transient way because the atoms are not bound in the other two dimensions. Twodimensional (2-d) and three-dimensional (3-d) optical lattices would be of greater interest because they trap atoms for longer times. To obtain them one needs a spatially modulated optical potential with wells in which atoms can be trapped. It should be noted that in the 2-d and 3-d cases, modulation of the optical potential results not only from polarization gradients (as in the case of Fig. 2) but also from intensity variations. Second, the light-field configuration should give rise to Sisyphus cooling. Third, in order to obtain long confinement times in a single potential well, the light polarization at the potential minima should be circular. Indeed the absorption of $\sigma^{+}$photon by an atom located in a $m_{\mathrm{a}}=+1 / 2$ potential well takes

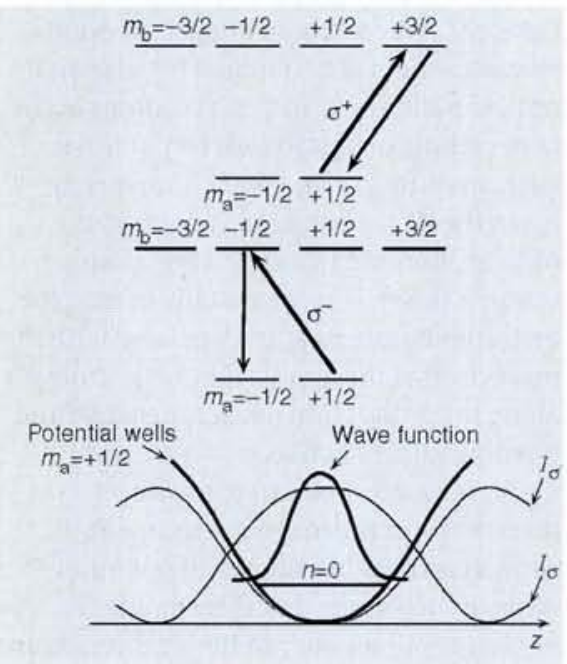

Fig. 4. Lifetimes of the vibrational levels. (a) Absorption of a $\sigma^{+}$-polarized photon by an atom in the $m_{\mathrm{a}}=+1 / 2$ sublevel transfers the atom to the $m_{\mathrm{b}}=+3 / 2$ state, from which is can only decay towards the $m_{\mathrm{a}}=+1 / 2$ state. The probability for an atom to undergo a change of vibrational state (b) during an absorption/spontaneous emission cycle of this type is very small owing to the strong spatial localization of its wavefunction (the Lamb-Dicke effect). It is small because the wings of the atomic wavefunction spread over a space domain where the intensity $/ \sigma$ of the $\sigma$ component of light has a finite value so an atom can escape from a $m_{a}=+1 / 2$ potential well and reach a $m_{\mathrm{a}}=-1 / 2$ well. (c) For the lowest vibrational levels where the atomic wavefunction is highly localized around the bottom of the optical potential well, the intensity $/ \sigma$ of $\sigma$ light seen by the atom, and hence the probability of leaving the potential well is very small. the atom to the $m_{\mathrm{b}}=+3 / 2$ level from which it can only decay towards the $m_{\mathrm{a}}=+1 / 2$ level (Fig. 4a). An atom can escape from a $m_{\mathrm{a}}=+1 / 2$ potential well and reach a $m_{\mathrm{a}}=$ $-1 / 2$ well because the wings of the atomic wavefunction spread over a space domain where the intensity of the $m_{\mathrm{a}}$-component of light differs from $o$ (Fig. 4 b). For the lowest vibrational levels, the wave-function is strongly confined near the potential minima (Fig. 4C), and the probability of absorbing a photon of the minority circular polarization in the well is considerably reduced [3].

The first 2-d potential that fulfilled all these conditions was realised experimentally by Hemmerich and Hänsch in Munich [7]. They used a light field which resulted from the superimposition of two linearly polarized standing waves propagating along orthogonal directions, the linear polarization being in the plane of propagation of the beams. By adjusting the relative phases of the beams, it was possible to obtain a field for which the intensity maxima coincided with points where the light was circularly polarized. When the lattice beams were tuned on the red side of the resonance condition, these points therefore corresponded to the minima of the optical potential, and oscillating atomic motion in these potential wells was observed.

The main difficulty of this approach lies in the fact that it is necessary to adjust the relative phase of the standing waves. This is because the polarization is circular at the maxima of intensity only when the relative phase equals $\pm \pi / 2$. If the relative phase changes, the polarization is no longer circular and the shape of the optical potential is distorted. As a result, the vibration structure disappears from the experimental spectra.

In order to understand the origin of this phase sensitivity, it is interesting to return to the 1 - $d$ case where the phenomenon is not found. Two counterpropagating travelling waves were used to produce the optical potential of Fig. 2. The phases of the waves determine the time origin and the position of the minima of the optical potential. Any variation of the relative phase between the two waves thus leads to a global translation of the potential along the propagation direction. The extension of this argument to the 2-d case shows that in order to achieve a 2-d optical potential for which the topography is insensitive to phase fluctuations, the maximum number of independent phases should be equal to three: one phase determines the time origin and the other two the position of the optical potential in the plane. One should thus use three beams for obtaining a phase independent potential shape in the 2 - $d$ case. In the of Hemmerich and Hänsch's experiment, there were two standing waves corresponding to four travelling waves. So there was a supplementary phase that required careful locking.

A three-beam experiment of the same type has been set up in Paris [8]. The three waves make an angle of $120^{\circ}$ to each other and have a linear polarization in their propagation plane. The resulting optical potential has intensity maxima at points where the light is circularly polarized. The potential wells are located on a hexagonal lattice and are alternatively $\sigma^{+}$and $\sigma$ (cover illustration). The occurrence of Raman components in the probe transmission spectra confirms the trapping of atoms in the potential wells and allows one to measure the vibration frequency in the wells.

It is well-known in solid-state physics that diffraction of an electromagnetic wave incident on ordered structures such as crystals only occurs in particular directions, called Bragg directions, which depend on the crystal geometry. These directions correspond to angles for which there is constructive interference between radiation scattered by individual atoms. Since the atoms trapped in the optical potential wells are located on a regular lattice, coherent diffraction should also occur in Bragg directions.

It is however possible to address the problem from a different point of view. In nonlinear optics, one expects that new light beams will be generated through nonlinear mixing processes in directions where all the atomic dipoles radiate in phase. These directions are those for which the phasematching condition is fulfiled. In the case where the generated field (wave vector $\boldsymbol{k}_{\mathrm{f}}$ ) has the same frequency as the incident field (wavevector $k_{\mathrm{i}}$ with $\left|k_{\mathrm{i}}\right|=\left|k_{\mathrm{f}}\right|$ ), the phasematching condition can be written as :

$$
k_{\mathrm{f}}=k_{\mathrm{i}}+p\left(k_{1}-k_{2}\right)+q\left(k_{1}-k_{3}\right)
$$

where $k_{1}, k_{2}$ and $k_{3}$ are the wavevectors of the lattice beams and $p$ and $q$ are integers. Because the atoms are bound by light, a consistent description of the optical lattice requires that these two methods for determining the direction of coherent scattering should coincide. The Bragg directions and the directions where the phase-matching condition is fulfilled are the same, and are both given by Eq. (1). 
Coherent emission in the Bragg directions was observed experimentally in Paris [8].

There is another way to interpret Eq. (1). When multiplied by $h$, the equation can be considered as being related to momentum conservation. The change of atomic momentum due to photon scattering from the $k_{i}$ towards the $k_{f}$ direction is $\left(\hbar \boldsymbol{k}_{\boldsymbol{i}}-\boldsymbol{h} \boldsymbol{k}_{f}\right)$, where $h \boldsymbol{k}_{i}$ is the photon momentum. In an elementary process of photon redistribution between the lattice beams, the atom absorbs a photon from a laser beam $k_{1}$ and emits a photon in another beam $k_{2}$ with a variation of momentum $\left(h \boldsymbol{k}_{1}-h \boldsymbol{k}_{2}\right)$. More generally, a redistribution process between lattice beams involves a change of momentum given by $p\left(\hbar \boldsymbol{k}_{1}-\hbar \boldsymbol{k}_{2}\right)+q\left(\hbar \boldsymbol{k}_{1}-\hbar \boldsymbol{k}_{3}\right)$. Eq. (1) thus means that the change of momentum of the scattered photon is exactly compensated for by a redistribution of photons between the lattice beams, so no momentum is transferred to the atoms.

\section{Many Possibilities}

The previous discussion indicates how a 3-d optical lattice could be built. Since two beams are necessary in 1- $d$, and three beams in 2-d, one expects that four beams are required in 3-d so as to establish an optical potential which is insensitive to phase fluctuations. In our first 3 -d experiment [8], the beams were aligned along the axis of a regular tetrahedron. As a result, the bound atoms were located on a body-centred cubic lattice. In a second set of experiments [9], we changed the angles between the lattice beams so that the atoms are located on an orthorhombic lattice. The dimensions of the elementary cell and the relative vibration frequencies of the atoms along the principal axis of the potential wells were modified by changing the angles between the beams.

The large variety of optical lattices that can be designed using four beams is easily understood. It is well known that Bragg scattering occurs in directions given by:

$$
k_{\mathrm{f}}-k_{\mathrm{i}}=K
$$

where $K$ is a reciprocal lattice vector. Comparing Eqs (1) and (2) - generalised to the 3 - $d$ case - shows that $\left(k_{1}-k_{2}\right),\left(k_{1}-k_{3}\right)$ and $\left(k_{1}-k_{4}\right)$ are basic vectors for the reciprocal lattice. The lattice is therefore completely determined by the directions of propagation of the laser beams. Conversely, it is possible to adjust the directions of the laser beams to generate lattices of various sizes and symmetries.

Another way of creating a 3 -d lattice is to use three orthogonal standing waves (i.e., six travelling waves) but in this case, two relative phases must be adjusted. Such lattices have been developed and studied by Hänsch, Hemmerich and their coworkers [10]. These always have the same cubic symmetry and the same size, but different bases can be obtained by adjusting the beam polarization.

\section{Filling of Optical Lattices}

The density of atoms in an optical lattice is an important issue. Up to now, the filling rate has always remained less than $10 \%$ which means that more than nine wells out of ten remain empty. From a practical point of view, this very lacuna structure originates from the density of the source of cold atoms used in today's experiments. However, it is not clear whether or not it is possible to increase the density significantly. Collisions between ultracold atoms may, for example, prevent two atoms from sharing the same potential well. These inter-atom interactions are especially pronounced because the atoms are located near points where they interact strongly with the field. This leads to a large induced dipole and consequently to a large dipole-dipole interaction.

A way to overcome this difficulty could be to trap atoms in lattices where the minima of the potential wells are located at points with a minimum amount of atomfield interaction. Recent calculations [11] and experiments [12] indicate that this new kind of optical lattices should be feasible.

\section{Current Developments}

Many interesting phenomena can be observed even at today's densities. Phillips and coworkers have measured a temperature of $1 \mu \mathrm{K}$ in an optical lattice. By slowly reducing the depth of the optical potential, the adiabatic expansion of the atomic wavefunction leads to still lower temperatures (700 $\mathrm{nK}[13]$ ).

We have studied in Paris the behaviour of the atoms in a 3 -d antiferromagnetic lattice in the presence of a static magnetic field. Because the field removes the degeneracy between the $\sigma^{+}$and $\sigma$ potential wells, a transfer of atoms from one type of well to the other generates a magnetization. From the relative population in the potential wells, it is possible to define a spin temperature which is close to (but not equal to) the kinetic temperature [14]. One important issue is to understand the long damping times which were observed. A natural time scale for processes occurring inside one well is the optical pumping time (the time a free atom takes to leave the initial Zeeman sublevel owing to its interaction with the incident beams) multiplied by a correction term [3] which is larger than unity, and increases as the atomic wavefunction becomes more and more confined inside the well (see Fig. 4c). Damping times some one order of magnitude larger than this time scale suggest that these times might be associated with the dynamics of an atom travelling over several lattice wells.

The observation of tunnelling between potential wells (i.e., the realisation of a real band structure with atoms), dimer formation, collective excitation, photonic bandgaps (i.e., structures in which light at some given frequency cannot propagate whatever the direction of incidence), and quasiperiodic optical lattices are among the subjects being considered for future experiments. More generally, optical lattices offer the possibility to scrutinise processes and concepts initially developed for condensed matter, but at totally different scales (for length, temperature, carrier mass) and in situations where the potential has no defects because it is created by light interference. The modification of the potential by the field radiated by the atoms bound in the lattice is also very interesting, and some first results have appeared [15]. Thus, the study of optical lattices has already generated some fundamental insight, for instance, quantization of the atomic motion in an optical potential and the light-induced localization of atoms on a regular lattice, but there is clearly much more in store.

\section{References}

[1] C. Cohen-Tannoudji, W.D. Phillips, Phys. Today 43 (1990) 33 .

[2] Y.Castin, J.Dalibard Europhys. Lett. 14 (1991) 761. [3] J.-Y.Courtois, G. Grynberg, Phys. Rev. A 46 (1992) 7060.

[4] P. Verkerk et al., Phys. Rev. Lett. 68 (1992) 3861.

[5] P. S. Jessen et al., Phys. Rev.Lett. 69 (1992) 49.

[6] B. Lounis et al., Europhys. Lett. 21 (1993) 13.

[7] A. Hemmerich, T.W. Hänsch, Phys. Rev. Lett. 70 (1993) 410.

[8] G. Grynberg, et al., Phys. Rev. Lett. 70 (1993) 2249.

[9] P. Verkerk et al., Europhys. Lett. 26 (1994) 171.

[10] A. Hemmerich, C.Zimmerman, T.W. Hänsch,

Europhys. Lett. 22 (1993) 89;A. Hemmerich et al.,

Europhys. Lett. 27 (1994) 427.

[11] G. Grynberg, J.-Y. Courtois, Europhys. Lett.

27 (1994) 41.

[12] A. Hemmerich, et al., Phys. Rev. Lett. 75 (1995) 37.

[13] A. Kastberg et al., Phys. Rev. Lett.74(1995) 171.

[14] D. Meacher et al., Phys. Rev. Lett.74 (1995) 1958.

[15] G. Birkl et al., Phys. Rev. Lett.74(1995) 2829. 